Y. C. Minh and E. F. van Dishoeck, eds.

\title{
Millimeter Observations of the Chemistry in Bipolar Outflows
}

\author{
Guido Garay \\ Departamento de Astronomía, Universidad de Chile, Casilla 36-D, \\ Santiago, Chile
}

\begin{abstract}
Millimeter wave observations of molecular lines provide a useful tool to study the chemistry of bipolar outflows. In this contribution we discuss recent observational results which have permitted to assess the chemical alterations of the ambient medium produced by the presence of bipolar collimated winds driven by young protostars. Discussed first are the results from molecular line surveys which have allowed to identify several species whose abundances in the outflow lobes are considerably enhanced with respect to that of the ambient medium. These enhancements are useful diagnostics of physical and chemical processes in the outflow lobes, such as sputtering of grain cores and/or desorption of grain mantles. Then we discuss the characteristics of the line profiles and of the spatial distribution of the emission in specific enhanced species, which provide information about the mechanisms responsible for the enhancements. In particular we give emphasis to the results derived from observations of two highly enhanced species, $\mathrm{SiO}$ and $\mathrm{CH}_{3} \mathrm{OH}$, which are observed to trace different components of the outflows.
\end{abstract}

\section{Introduction}

A wealth of observations have shown that bipolar molecular outflows are common phenomena in the process of star formation (see reviews by Bachiller \& GómezGonzález 1992; Bachiller 1996). During their earliest phase of evolution, young stellar objects generate a fast, well collimated bipolar wind which is thought to drive away the original angular momentum of the collapsing cloud core. As the high velocity gas interacts with and sweeps up the surrounding ambient gas and dust, shock waves are driven into the quiescent ambient medium giving rise to the molecular outflows.

Theoretical calculations show that shock waves which compress and heat the gas cause substantial effects on the chemistry of the originally quiescent material. The characteristics depend on whether the shocks are of the jump (J) or continuous (C) type (see review by Draine \& McKee 1993), which in turn depends on the shock velocity, magnetic field strength, and fractional ionization in the preshock gas. In high velocity J-type shocks $\left(V_{S}>50 \mathrm{~km} \mathrm{~s}^{-1}\right)$ the temperature of the gas is high enough $\left(10^{4}-10^{5} \mathrm{~K}\right)$ to dissociate all molecules, which are reformed in a warm zone in the wake of the shock (Hollenbach \& McKee 1989; Neufeld \& Dalgarno 1989). Thermal sputtering of grain cores is expected to inject a high abundance of refractory elements such as Si and Fe. In lower 
speed $\left(V_{S}<30 \mathrm{~km} \mathrm{~s}^{-1}\right)$ C-type shocks the temperature of the gas is increased to only $\sim 10^{3} \mathrm{~K}$, and the fluid remains molecular. Several endothermic reactions proceed rapidly in the warm gas producing species, such as $\mathrm{OH}, \mathrm{H}_{2} \mathrm{O}, \mathrm{H}_{2} \mathrm{~S}$, which are otherwise rare in the ambient medium. In addition, $\mathrm{C}$-shocks are expected to introduce a variety of refractory and volatile species on the gas phase via non-thermal sputtering of grain cores and ice mantles (Flower \& Pineau des Forêts 1995; Tielens 1999).

Outflows from young stars are then expected not only to affect considerably the physical properties of the surrounding gas but to produce significant alterations in the chemistry of the ambient medium. They create high temperature shocks and lower-temperature turbulent regions in which the icy mantles and refractory material can be returned to the gas. Thus, studies of the chemical composition of bipolar molecular outflows should permit one to investigate their structure. In addition, since shocks are transient phenomena, it would be possible to use molecules with enhanced abundances as temporal indicators and hence as signposts of the evolutionary state of the outflow.

Most of the imaging of molecular outflows has so far been made in lines of $\mathrm{CO}$ which is the most sensitive tracer of the mass in the outflow. The observed morphologies can be classified in two categories: "classical", which is characterized by a moderate degree of collimation, and "highly collimated". The majority of the outflows are of the classical type, while few are highly collimated (André et al. 1990; Bachiller et al. 1990; Lada \& Fich 1996). The two classes are thought to mark different stages of outflow life, with the highly collimated outflows being associated with the youngest protostars (or Class 0 objects). Carbon monoxide is not, however, the most adequate probe to assess chemical changes in bipolar outflows. $\mathrm{CO}$ emission is ubiquitous and therefore studies of the outflowing gas are usually hampered by confusion produced by different components along the line of sight. In addition, the CO chemistry is relatively insensitive to shocks (Mitchell 1984). In this contribution we discuss recent results regarding the chemical composition and structure of bipolar outflows, derived from mm-wave observations. In $\S 2$ we summarize the results from molecular line surveys toward outflow lobes which have allowed to identify several species whose abundances are considerably enhanced with respect to that of the ambient medium. The characteristics of the line profiles and of the spatial distribution of the emission in specific enhanced species are presented in $\S 3$ and $\S 4$, respectively. In $\S 5$ we discuss mechanisms responsible for the abundance enhancements. Finally, in $\S 6$ we discuss the role of jets in determining the chemical characteristics of bipolar outflows. A thorough review of the chemistry of bipolar molecular outflows has been recently presented by van Dishoeck \& Blake (1998).

\section{Molecular Line Surveys}

Spectral surveys of line emission from bipolar outflows are crucial since they permit to identify the molecular species that are produced selectively behind shock fronts. They also provide insights of shock chemistry, allowing to assess the importance of processes such as endothermic reactions, sublimation of ices, and disruption of dust grain cores. Although more than 250 bipolar outflows are currently known from observations of $\mathrm{CO}$ and its isotopes (Wu, Huang, \& $\mathrm{He}$ 

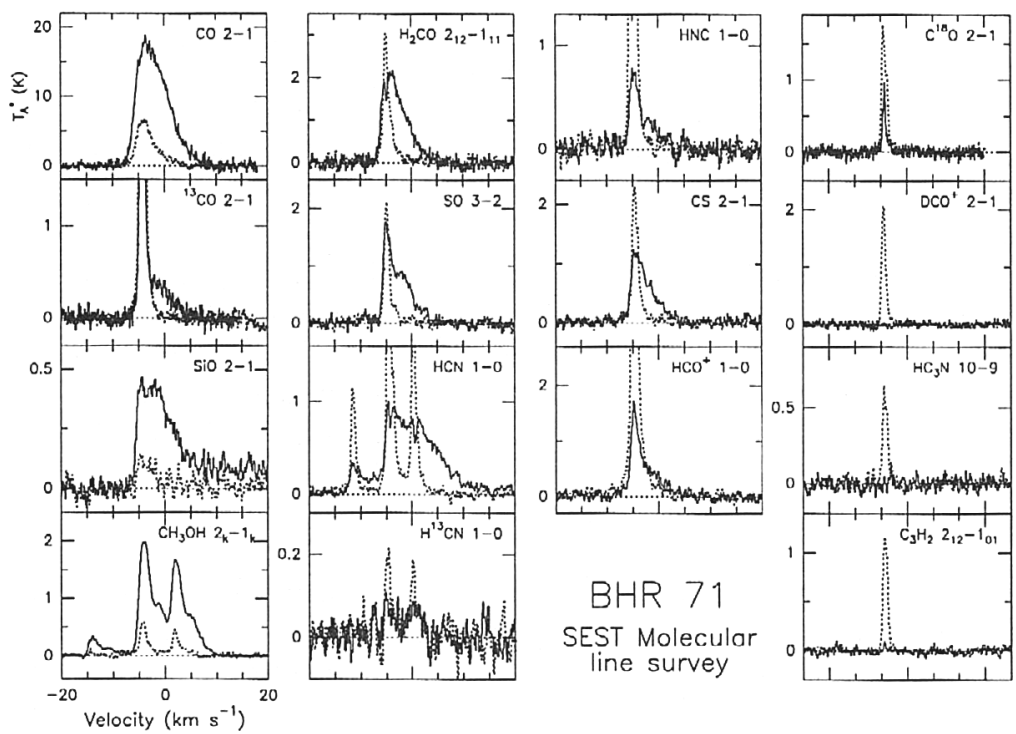

Figure 1. Line profiles observed toward the redshifted lobe (continuous lines) and central position (dotted lines) of the BHR71 outflow.

1996), only a handful of them have been inspected in a number of other chemical species. The sample includes four bipolar outflows associated with low-mass YSO's (IRAS 05338-0624, McMullin, Mundy, \& Blake 1994; NGC1333/IRAS4, Blake et al. 1995; L1157, Bachiller \& Pérez-Gutiérrez 1997; BHR71, Bourke \& Garay 2000) and two outflows associated with intermediate to high mass YSO's (OMC-1/IRc2, Blake et al. 1987; NGC 2071, Girart et al. 1999 and references therein). The spectral line surveys show that the abundances of some refractory species (such as $\mathrm{SiO}$ ) and some volatile mantle species (such as $\mathrm{CH}_{3} \mathrm{OH}$ ) are substantially enhanced in the outflowing material. Nearly 15 molecular species, not including isotopic varieties, have been detected toward the lobes of bipolar outflows from mm-wave observations, including: $\mathrm{CO}, \mathrm{CN}, \mathrm{CS}, \mathrm{SO}, \mathrm{SiO}, \mathrm{SO}_{2}$, $\mathrm{H}_{2} \mathrm{~S}, \mathrm{HCN}, \mathrm{HNC}, \mathrm{HCO}^{+}, \mathrm{H}_{2} \mathrm{CO}, \mathrm{H}_{2} \mathrm{CS}, \mathrm{HC}_{3} \mathrm{~N}$, and $\mathrm{CH}_{3} \mathrm{OH}$. Note that other molecular species, such as $\mathrm{H}_{2}, \mathrm{H}_{2} \mathrm{O}, \mathrm{OH}$, and $\mathrm{NH}_{3}$, which do not emit at mmwavelengths, have also been detected toward outflow lobes. The spectral surveys also show that some molecules that are relatively abundant in the quiescent medium are not present (below detection limits) in the shocked region (e.g. $\left.\mathrm{C}_{3} \mathrm{H}_{2}, \mathrm{~N}_{2} \mathrm{H}^{+}, \mathrm{H}^{13} \mathrm{CO}^{+}, \mathrm{DCO}^{+}\right)$. These results are illustrated in Fig. 1 which shows a sample of molecular line profiles taken toward the redshifted lobe and central position of the BHR71 bipolar outflow.

In order to establish whether the above results are typical of bipolar outflows, some studies concentrate on observations of a large number of objects in a single species. Chernin, Masson, \& Fuller (1994) searched for sulfur monoxide (SO) emission in three transitions $\left(J_{N}=2_{3}-1_{2}, 3_{2}-2_{1}, 6_{5}-5_{4}\right)$ toward nine regions containing molecular outflows. High velocity wing $\mathrm{SO}$ emission was detected in about half of the outflows, typically toward the outer parts of the CO lobes, and only in the $6_{5}-5_{4}$ transition. Chandler \& Richer (1997) searched for $\mathrm{SiO}$ emission, in the $J=5-4$ line, toward 15 molecular outflows associated 
with protostars in different stages of evolution. They found that $\mathrm{SiO}$ emission is seen preferentially toward the youngest (class 0 ) protostars ( $50 \%$ of detections), no $\mathrm{SiO}$ emission was detected from outflows associated with class II sources. Codella, Bachiller, \& Reipurth (1999) searched for SiO emission toward 17 star forming regions, detecting emission in $\sim 25 \%$ of the regions containing HerbigHaro objects and in $\sim 75 \%$ of the regions containing molecular outflows. Avery \& MacLeod (2000) searched for methanol line emission in approximately 40 outflows, detecting emission in about $25 \%$ of them. These studies show that the detection of emission in refractory species $(\mathrm{SiO})$ and/or volatile mantle species species $\left(\mathrm{CH}_{3} \mathrm{OH}\right)$ from $\mathrm{CO}$ bipolar outflows is not common (1 of 4). The detection rate increases to $\sim 50 \%$ for $\mathrm{CO}$ outflows associated with class 0 protostars.

\section{Line Profiles}

The line profiles of molecular species for which the outflow emission is much stronger than that from the narrow, quiescent ambient gas component provide a powerful tool for investigating the kinematics of the outflowing molecular gas. Accordingly, the number of bipolar outflows observed in molecular species other than $\mathrm{CO}$ has increased significantly over the last few years. The observed line profiles exhibit a variety of shapes, and are different for different molecules (see Fig. 1). In some species (e.g. $\mathrm{CS}, \mathrm{HCO}^{+}$) the emission from the outflowing gas appears as a broad feature superposed upon a strong narrow feature from the quiescent ambient gas. In others (e.g. $\left.\mathrm{CH}_{3} \mathrm{OH}, \mathrm{HCN}\right)$ the intensity of the outflow emission feature is comparable to that of the narrow quiescent component. Finally, in a few species, most notable $\mathrm{SiO}$ and $\mathrm{SO}$, the emission from the outflowing material completely dominates the emission spectra. In addition, the observed line profiles suggest the presence of two distinct outflow components: a high velocity component and a low velocity component.

In the following we will focus on the observed profiles in $\mathrm{SiO}$ whose emission arises mainly from outflowing gas and therefore is one of the principal diagnostics of shocks. Figure 2 presents a sample of $\mathrm{SiO}$ profiles observed toward a few bipolar outflows. Two broad classes of line shapes, referred to as Type I and Type II, can be distinguished. Type I profiles are characterized by exhibiting a strong emission feature at velocities that are considerably displaced from the ambient cloud velocity, a steep decrease in intensity toward higher outflow velocities and a slower decline toward the ambient cloud velocity (e.g. L1448-mm). Type II profiles, on the other hand, are characterized by showing a peak at a radial velocity close to the ambient cloud velocity, a gradual decrease in intensity from the peak toward higher outflow velocities, and a rapid decline toward the ambient cloud velocity. Some Type II profiles show emission from only a low outflow velocity component (e.g. SMM4) whereas others show emission from both a high and a low outflow velocity component (e.g. NGC2071-blue). Most of the observed $\mathrm{SiO}$ profiles are of Type II, suggesting that in most lobes a small fraction of the molecular gas is accelerated to shock velocities while the majority remains close to the ambient cloud velocity. The different types of profiles most likely reflect differences in the physical conditions of the ambient gas, driving source, and mechanism of shock excitation.

Is shock type the main agent responsible for the difference in observed profiles? An observer of a shock moving with speed $V_{S}$, at rest with respect 


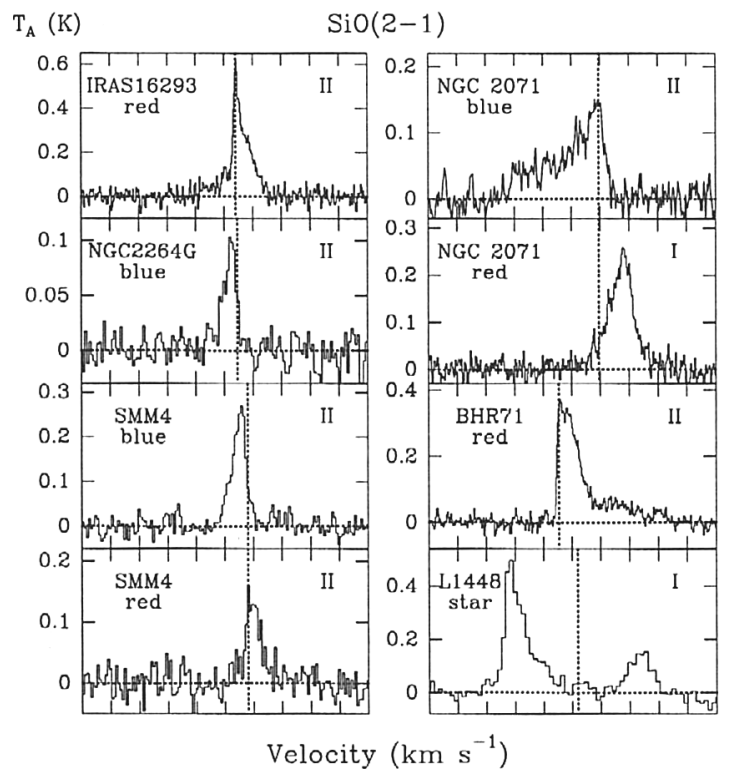

Figure 2. Line profiles of the $\mathrm{SiO}$ emission from a sample of bipolar outflows. The velocity scale is from -50 to $50 \mathrm{~km} \mathrm{~s}^{-1}$, except for L1448 which is from -125 to $125 \mathrm{~km} \mathrm{~s}^{-1}$ (vertical dash line: ambient cloud velocity).

to the pre-shock gas, will see broad lines with a significant contribution from velocities considerably lower than $V_{S}$ for a C-type shock and relatively narrow lines at velocities of $\sim \pm V_{S}$ for a J-type shock (cf. Hollenbach 1997). Thus one might expect that Type I profiles are associated with J-type shocks and Type II profiles with C-type shocks. The situation may not be clear cut, however. In the planar C-type shock models of Schilke et al. (1997) the main contribution to the column density of $\mathrm{SiO}$ arises from regions where the difference in velocity between the neutrals and ions and charged particles is the largest, hence they predict that the line shape of the $\mathrm{SiO}$ emission should exhibit Type I profiles. It may be argued that if a more realistic bow shock geometry is considered, where a range of shock speeds is present, the profiles may be of Type II. Models of emission line profiles from C-type bow shocks show in fact that the peak is close to the velocity of the pre-shock gas (Smith \& Brand 1990). However, they also show that significant overlapping of redshifted and blueshifted emission should be observed over a broad range of view angles (Stone \& Norman 1993), which is not a common property of the observed bipolar outflows. Therefore, line profiles as currently modelled do not show good agreement with the observations.

Finally, it is worth mentioning that a narrow and bright $\mathrm{SiO}$ component at the velocity of the quiescent ambient gas is seen in a few bipolar outflows (Lefloch et al. 1998; Garay et al. 1998; Codella et al. 1999). Since SiO emission from cold dark clouds is rarely detected, the origin of this low velocity $\mathrm{SiO}$ emission is not clear. It may arise from ambient gas that has been heated by the UV photons and radiation produced by the YSO and/or shocks. 


\section{Spatial Distribution}

Studies of the parcels of gas where enhanced molecular emission occurs have a great potential in determining the nature of the interaction of a collimated wind and/or jet with its environment. The spatial distribution of the gas at different velocities offers an important clue to the acceleration mechanism. In particular, understanding how the momentum is transfered by the jet to the surrounding gas can provide valuable information about the jet itself.

Mapping of the line emission in molecules, other than $\mathrm{CO}$, have been carried out toward few outflows: L1448-mm (Bachiller, Martín-Pintado, \& Fuente 1991, Guilloteau et al. 1992; Dutrey, Guilloteau, \& Bachiller 1997); NGC 1333/IRAS 2 (Bachiller et al. 1998); NGC 1333/IRAS 4 (Lefloch et al. 1998); IRAS 03282+3035 (Bachiller et al. 1994); L1157 (Mikami et al. 1992; Zhang et al. 1995; Avery \& Chiao 1996; Gueth, Guilloteau, \& Bachiller 1998); HH 211 (Chandler \& Richer 1997); BHR71 (Garay et al. 1998), and in few molecular species. Hereafter, we concentrate the discussion on the spatial distribution of the $\mathrm{SiO}$ emission from three objects, L1448-mm, IRAS 03282+3035, and BHR71, which present clear differences in the location of the shock enhanced emission relative to that of the $\mathrm{CO}$ emission.

L1448-mm is the prototype of outflows in which the $\mathrm{SiO}$ emission arises from a highly collimated bipolar jet-like structure located close to the driving source (Guilloteau et al. 1992). Whereas the CO outflow shows a well collimated bipolar structure with a total extent of $\sim 0.4 \mathrm{pc}$ (Bachiller et al. 1990), the SiO jet-like structure extends only up to a distance of $\sim 0.01 \mathrm{pc}$ from the central exciting source. This inner $\mathrm{SiO}$ jet consists of a chain of well aligned high velocity clumps moving with radial velocities of up to $70 \mathrm{~km} \mathrm{~s}^{-1}$ relative to the ambient gas. The knot-like structure along the jet may indicate an intermittent nature of the outflow. The enhancement along the axes of the molecular outflow suggests that the highly collimated $\mathrm{SiO}$ features correspond to turbulent layers entrained around YSO jets. The alternative explanation is that the jets are intrinsically molecular. IRAS $03282+3035$ is an example of the type of object in which the $\mathrm{SiO}$ emission arises from material located at the end of $\mathrm{CO}$ outflow lobes (Bachiller et al. 1994). This distribution is suggestive of a bow shock generated by a supersonic jet as it entrains ambient molecular material. Finally, BHR71 is representative of bipolar outflows in which the spatial distribution of the $\mathrm{SiO}$ emission is widespread, similar in extent to that of the CO emission (Garay et al. 1998). This type of morphology suggests that the chemical enhancement in these sources takes place in a shell-like structure produced by the dynamical interaction between the ambient cloud and an underlying wide-angled wind or wind driven shell. Alternatively, the lobes may consist of expanding cavities that are evacuated by the propagation of large bow shocks (Gueth, Guilloteau, \& Bachiller 1996).

\section{Enhancements}

The strong molecular emission observed in selected species toward the lobes of outflows is most likely due to a large increase in their abundances with respect to that of the ambient medium. There are, however, other contributing factors such 
as high excitation temperatures and high densities and hence the determination of molecular abundances from the spectral line data is not straightforward (see van Dishoeck \& Hogerheijde 1999). Table 1 presents a summary of the abundances of several molecular species, relative to that of $\mathrm{CO}$, in five outflows and for comparison in two dark clouds. Most notable is the enhancement of $\mathrm{SiO}$ whose abundance in outflows is several orders of magnitude larger than that in dark clouds.

What are the processes responsible for the abundance enhancements? The intimate association of outflow lobes and shocks suggests that shocks play an essential role in the production of these molecules (Bachiller 1996). Shocks can raise the gas temperature and drive many chemical reactions which are inefficient at ambient cloud temperatures. They can also partially destroy dust grains leading to the injection of several absorbed atoms and molecules from the grain surface into the gas phase. Shock chemistry involves gas phase processes at high temperatures in a medium enriched by species released from grains by shock processing, sublimation of ices and disruption of dust grain cores. In the following we discuss the production of selected, highly enhanced chemical species in collimated bipolar outflows, such as $\mathrm{SiO}$ and $\mathrm{CH}_{3} \mathrm{OH}$.

In cold dark molecular clouds the abundance of $\mathrm{SiO}$ is low $\left(\left[\mathrm{SiO} / \mathrm{H}_{2}\right]<10^{-11}\right)$ due to the considerable depletion of silicon from the gas phase into grains. It has been suggested that $\mathrm{SiO}$ in the outflow lobes is formed in shocked layers, in which the shock waves have partially destroyed the grains ejecting silicon into the gas phase which is then oxidized in the gas phase (Martín-Pintado, Bachiller, \& Fuente 1992). This suggestion is amply supported by theoretical studies which show that shocks can, depending on their velocity, release Si from grain cores or Si-bearing material from grain mantles into the gas phase (Schilke et al. 1997; Caselli, Hartquist, \& Havnes 1997). Once Si is in the gas phase in the warm post-shock region, it will react with $\mathrm{OH}$ and $\mathrm{O}_{2}$ to form $\mathrm{SiO}$ (Iglesias \& Silk 1978; Hartquist et al. 1980; Neufeld \& Dalgarno 1989). Since in grain cores $\mathrm{Si}$ is found in the form of refractory material, which is difficult to destroy, significant grain core destruction requires shock velocities $>25 \mathrm{~km} \mathrm{~s}^{-1}$. Thus the zones of enhanced $\mathrm{SiO}$ emission would mark the location of directly shocked material at high velocities. Since grain destruction is more efficient as the shock velocity increases, one expects to find an increase in the enhancement of the $\mathrm{SiO}$ abundance at higher flow velocities. Note, however, that these models predict

Table 1. Molecular abundances relative to $\mathrm{CO}$.

\begin{tabular}{|c|c|c|c|c|c|c|c|c|}
\hline Source & {$\left[\frac{c s}{c o}\right]$} & {$\left[\frac{\mathrm{so}}{\mathrm{CO}}\right]$} & {$\left[\frac{\mathrm{SiO}}{\mathrm{CO}}\right]$} & {$\left[\frac{\mathrm{HCN}}{\mathrm{CO}}\right]$} & {$\left[\frac{\mathrm{HCO}+}{\mathrm{CO}}\right]$} & {$\left[\frac{\mathrm{H}_{2} \mathrm{CO}}{\mathrm{CO}}\right]$} & {$\left[\frac{\mathrm{CH}_{3} \mathrm{OH}}{\mathrm{CO}}\right]$} & Ref. \\
\hline \multicolumn{9}{|c|}{ Outflows } \\
\hline $\begin{array}{l}\text { NGC1333-4 } \\
\text { IRAS05338 } \\
\text { BHR71 } \\
\text { L1157 } \\
\text { OMC-1 }\end{array}$ & $\begin{array}{l}1 \times 10^{-3} \\
1 \times 10^{-4} \\
6 \times 10^{-5} \\
2 \times 10^{-3} \\
2 \times 10^{-4}\end{array}$ & $\begin{array}{c}2 \times 10^{-3} \\
3 \times 10^{-4} \\
- \\
2 \times 10^{-3} \\
4 \times 10^{-3}\end{array}$ & $\begin{array}{l}7 \times 10^{-5} \\
3 \times 10^{-5} \\
9 \times 10^{-6} \\
6 \times 10^{-4} \\
2 \times 10^{-4}\end{array}$ & $\begin{array}{c}4 \times 10^{-4} \\
2 \times 10^{-4} \\
- \\
3 \times 10^{-3} \\
2 \times 10^{-3}\end{array}$ & $\begin{array}{c}5 \times 10^{-5} \\
- \\
5 \times 10^{-6} \\
5 \times 10^{-4} \\
-\end{array}$ & $\begin{array}{c}2 \times 10^{-3} \\
- \\
- \\
3 \times 10^{-3} \\
3 \times 10^{-4}\end{array}$ & $\begin{array}{c}3 \times 10^{-2} \\
3 \times 10^{-3} \\
2 \times 10^{-3} \\
1 \times 10^{-1} \\
-\end{array}$ & $\begin{array}{l}1 \\
2 \\
3 \\
4 \\
5\end{array}$ \\
\hline \multicolumn{9}{|c|}{ Dark clouds } \\
\hline $\begin{array}{l}\text { TMC-1 } \\
\text { L134N } \\
\end{array}$ & $\begin{array}{l}1 \times 10^{-4} \\
9 \times 10^{-6}\end{array}$ & $\begin{array}{l}6 \times 10^{-5} \\
3 \times 10^{-4} \\
\end{array}$ & $\begin{array}{l}<3 \times 10^{-8} \\
<5 \times 10^{-8} \\
\end{array}$ & $\begin{array}{l}1 \times 10^{-4} \\
5 \times 10^{-5}\end{array}$ & $\begin{array}{l}9 \times 10^{-5} \\
1 \times 10^{-4}\end{array}$ & $3 \times \overline{10^{-4}}$ & $\begin{array}{l}3 \times 10^{-5} \\
4 \times 10^{-5}\end{array}$ & $\begin{array}{l}3 \\
1 \\
\end{array}$ \\
\hline
\end{tabular}

References: ${ }^{1}$ Blake et al. 1995; ${ }^{2}$ McMullin, Mundy, \& Blake 1994; ${ }^{3}$ Garay et al. 1998;

${ }^{4}$ Bachiller \& Pérez-Gutiérrez 1997; ${ }^{5}$ Blake et al. 1987 
that the $\mathrm{SiO}$ emission should be observed at velocities near the shock velocity, which for efficient sputtering should be greater than $20 \mathrm{~km} \mathrm{~s}^{-1}$. Hence they fail, at least in their simple form, to explain Type II line profiles which are commonly observed in $\mathrm{SiO}$ outflows.

Methanol is another species whose gas phase abundance in outflow lobes is greatly enhanced with respect to that of the ambient medium (see Table 1). Tielens \& Allamandola (1987) suggested that the large abundance of methanol, one of the most abundant ices on grain surfaces, may arise from the evaporation of ice mantles. This is supported by the work of Blake et al. (1991) who found that at dust temperatures of $\sim 120 \mathrm{~K}$ interstellar ice grains selectively eject $\mathrm{CH}_{3} \mathrm{OH}$. Also grain-grain collisions induced by velocity shear zones surrounding the outflow axis may transiently desorb the grain mantles, resulting in large abundance enhancements of selected species. The volatile species can only survive sputtering or desorption of grain mantles at low shock velocities $\left(V_{s} \leq 10\right.$ $\mathrm{km} \mathrm{s}^{-1}$ ). Thus, the rich chemistry in species like $\mathrm{CH}_{3} \mathrm{OH}, \mathrm{HCN}$, and $\mathrm{H}_{2} \mathrm{CO}$, is thought to be driven by the evaporation of ice mantles due to heating by low velocity shocks. This is consistent with the observations which show that the outflow emission from volatile species is close to the ambient cloud velocity. The evaporated molecules subsequently drive a rapid gas phase chemistry leading to complex organic molecules.

\section{Jets or Wide-angle Winds ?}

The mechanism of formation of molecular outflows, which are thought to be made up of swept-up ambient gas rather than original ejecta from the driving source, is still a matter of current debate. Two main types of physical agents have been proposed: a wide angle stellar wind (e.g. Cabrit \& Bertout 1986; Shu et al. 1991) and a highly collimated wind or jet (e.g. Masson \& Chernin 1993; Raga et al. 1993). In the later case the molecular outflow may correspond to either a bow-shock created by an internal jet working surface (e.g. Raga \& Cabrit 1993) or a turbulent flow of molecular gas entrained at the sides of a fast jet (e.g. Cantó \& Raga 1991). The spatial distribution and the velocity structure of the molecular emission should give insight into how collimated outflows from young stars interact with their surroundings and help to discriminate between different models.

The observations of the shock enhanced species show that there is a relationship between the velocity of the molecular emission and its morphology. The extremely collimated jet-like $\mathrm{SiO}$ structures, which emerge from the protostellar condensation and are located close to the axis of the low velocity $\mathrm{CO}$ cavity, are invariably associated with high velocity $\mathrm{SiO}$ emission. This suggests that the highly collimated $\mathrm{SiO}$ features correspond to turbulent boundary layers that grow along the body of a supersonic jet (Cantó \& Raga 1991). The SiO structures located at the end of the CO outflow axis are, on the other hand, usually associated with moderate flow velocities, showing emission line profiles of Type II. This suggests that the $\mathrm{SiO}$ enhancement in these regions is confined to the immediate vicinity of a bow shock generated by a fast jet as it entrains ambient molecular material. The two types of observed spatial-velocity relationship described above are, thus, thought to be linked to the presence of an underlying 
supersonic jet ejected from the central star/disk system. Finally, in the sources in which the $\mathrm{SiO}$ is widely spread within the $\mathrm{CO}$ lobes the $\mathrm{SiO}$ outflow velocities are low, close to the ambient cloud velocity. The $\mathrm{SiO}$ emission is thought to comprise mostly of swept up ambient gas and traces the inner edge of outflow cavities. Thus, the chemical enhancement in these sources is likely to occur in a shocked shell-like structure produced by the dynamical interaction between the ambient cloud and an underlying wide-angle wind or wind driven shell.

In summary, the mm-wave observations discussed in this contribution provide clear evidence that the chemical characteristics of bipolar outflows strongly depend on their degree of collimation, hence on the characteristics of the underlying driving agent (jet or wide angle wind), and on the class of central source, hence on the outflow age. Outflows associated with class I and class II YSO's and outflows with low collimation, which are thought to be driven by wide angle winds, usually do not show emission in enhanced lines. On the other hand, the chemistry is most affected for bipolar outflows associated with class 0 objects. These outflows are characterized by being highly collimated and having extremely high velocities, and are thought to contain an embedded jet. Where the jet interacts with the ambient medium it creates external shocks at the ends or the sides of an outflow cavity and accelerates the surrounding gas triggering a shock chemistry. Enhancement occurs either at the leading edge of the propagating jet, where the jet drives a strong bow shock into the ambient medium (prompt entrainment), or along the jet far behind the leading shock wave (steady state entrainment).

Acknowledgments. G.G. gratefully acknowledges support from a Chilean Presidential Science Fellowship and from Fondecyt Project 1980660.

\section{References}

André, Ph., Martín-Pintado, J., Despois, D., \& Montmerle, T. 1990, A\&A, 236, 180 Avery, L.W. \& Chiao, M. 1996, ApJ, 463, 642

Avery, L.W. \& McLeod, J.M. 2000, in preparation

Bachiller, R. 1996, ARA\&A, 34, 111

Bachiller, R., Cernicharo, J., Martín-Pintado, J., Tafalla, M., \& Lazareff, B. 1990, A\&A, 231, 174

Bachiller, R., Codella, C., Colomer, F., Liechti, S., \& Walmsley, C.M. 1998, A\&A, 335, 266

Bachiller, R. \& Gómez-González, J. 1992, Astron. Astrophys. Rev., 3, 257

Bachiller, R., Martín-Pintado, J., \& Fuente, A. 1991, A\&A, 243, L21

Bachiller, R. \& Pérez Gutiérrez, M., 1997, ApJ, 487, L93

Bachiller, R., Terebey, S., Jarrett, T., et al. 1994, ApJ, 437, 296

Blake, D., Allamandola, L., Sandford, S., Hudgins, D., \& Freund, F. 1991, Science, 254, 548

Blake, G.A., Sandell, G., van Dishoeck, E.F., Groesbeck, T. D., Mundy, L.G., \& Aspin, C. 1995, ApJ, 441, 689

Blake, G.A., Sutton, E.C., Masson, C.R., \& Phillips, T.G. 1987, ApJ, 315, 621

Bourke, T.L. \& Garay, G. 2000, in preparation

Cabrit, S. \& Bertout, C. 1986, ApJ, 307, 313

Cantó, J. \& Raga, A. 1991, ApJ, 372, 646

Caselli, P., Hartquist, T.W., \& Havnes, O. 1997, A\&A, 322, 296 
Chandler, C.J. \& Richer, J.S. 1997, Poster of IAU Symp. 182, Herbig-Haro Objects and the Birth of Low Mass Stars, eds. F. Malbet \& A. Castets, 76 Chernin, L.M., Masson, C.R., \& Fuller, G.A. 1994, ApJ, 436, 741

Codella, C., Bachiller, R., \& Reipurth, B. 1999, A\&A, 343, 585

Draine, B.T. \& McKee, C.F. 1993, ARA\&A, 31, 373

Dutrey, A., Guilloteau, S., \& Bachiller, R. 1997, A\&A, 325, 758

Flower, D.R. \& Pineau des Forêts, G. 1995, MNRAS, 275, 1049

Garay, G., Köhnenkamp, I., Bourke, T.L., Rodríguez, L.F., \& Lehtinen, K.K. 1998, ApJ, 509, 768

Girart, J.M., Ho, P.T.P., Rudolph, A.L., et al. 1999, ApJ, 522,

Gueth, F., Guilloteau, S., \& Bachiller, R. 1996, A\&A, 307, 891 1998, A\&A, 333, 287

Guilloteau, S., Bachiller, R., Fuente, A., \& Lucas, R. 1992, A\&A, 265, L49

Hartquist, T.W., Oppenheimer, M., \& Dalgarno, A. 1980, ApJ, 236, 182

Hollenbach, D. 1997, in IAU Symp. 182, Herbig-Haro Flows and the Birth of Low Mass Stars, eds. B. Reipurth \& C. Bertout (Dordrecht: Reidel), 181

Hollenbach, D. \& McKee, C.F. 1989, ApJ, 342, 306

Iglesias, E.R. \& Silk, J. 1978, ApJ, 226, 851

Lada, C.J. \& Fich, M. 1996, ApJ, 459, 638

Lefloch, B., Castets, A., Cernicharo, J., \& Loinard, L. 1998, ApJ, 504, L109

Martín-Pintado, J., Bachiller, R., \& Fuente, A. 1992, A\&A, 254, 315

Masson, C.R. \& Chernin, L.M. 1993, ApJ, 414, 230

McMullin, J.P., Mundy, L.G., \& Blake, G.A. 1994, ApJ, 437, 305

Mikami, H., Umemoto, T., Yamamoto, S., \& Saito, S. 1992, ApJ, 392, L87

Mitchell, G.F. 1984, ApJS, 54, 81

Neufeld, D.A. \& Dalgarno, A. 1989 ApJ, 340, 869

Raga, A.C. \& Cabrit, S. 1993, A\&A, 278, 267

Raga, A.C., Cantó, J., Calvet, N., et al. 1993, A\&A, 276, 539

Schilke, P., Walmsley, C.M., Pineau des Forêts, G., \& Flower, D.R. 1997, A\&A, 321,293

Shu, F.H., Ruden, S.P., Lada, C.J., \& Lizano, S. 1991, ApJ, 370, L31

Smith, M.D. \& Brand, P.W.J.L. 1990, MNRAS, 245, 108

Stone, J.M. \& Norman, M.L. 1993, ApJ, 413, 210

Tielens, A.G.G.M. 1999, Formation and Evolution of Solids in Space, eds. J.M.

Greenberg \& A. Li (Dordrecht: Kluwer), 331

Tielens, A.G.G.M. \& Allamandola, L.J. 1987, in Physical Processes in Interstellar Clouds, eds. G.E. Morfill \& M. Scholer (Dordrecht: Reidel), 333

van Dishoeck, E.F. \& Blake, G.A. 1998, ARA\&A, 36, 317

van Dishoeck, E.F. \& Hogerheijde, M.R. 1999, Physics of Star Formation and

Early Stellar Evol. II, eds. C.J. Lada \& N.D. Kylafis (Dordrecht:Kluwer)

Wu, Y., Huang, M., \& He, J. 1996, A\&AS, 115, 283

Zhang, Q., Ho, P.T.P., Wright, M.C.H., \& Wilner, D.J. 1995, ApJ, 451, L71

\section{Discussion}

T. Millar: Comment. It is dangerous to assume that all methanol enhancements observed in outflows come from evaporation of ice mantles. No one has ever included methanol formation in any shock model - we do not know whether shocks can produce methanol, nor whether they can destroy methanol. 